\title{
Licochalcone B induces apoptosis of human oral squamous cell carcinoma through the extrinsic- and intrinsic-signaling pathways
}

\author{
HANA OH $^{1 *}$, GOO YOON $^{1 *}$, JAE-CHEON SHIN $^{2}$, SEON-MIN PARK $^{2}$, SEUNG-SIK CHO $^{1}$, JIN HYOUNG CHO $^{3}$, \\ MEE-HYUN LEE ${ }^{4}, \mathrm{KANGDONG} \mathrm{LIU}^{4,5}$, YOUNG SIK CHO ${ }^{6}$, JUNG-IL CHAE ${ }^{3}$ and JUNG-HYUN SHIM ${ }^{1}$ \\ ${ }^{1}$ Department of Pharmacy, College of Pharmacy and Natural Medicine Research Institute, \\ Mokpo National University, Jeonnam 534-729; ${ }^{2}$ Pohang Center for Evaluation of Biomaterials, Pohang, \\ Gyeongbuk 790-834; ${ }^{3}$ Department of Dental Pharmacology, School of Dentistry and Institute of Oral Bioscience, BK21 Plus, \\ Chonbuk National University, Jeonju 651-756, Republic of Korea; ${ }^{4}$ The China-US (Henan) Hormel Cancer Institute; \\ ${ }^{5}$ The School of Basic Medical Sciences, Zhengzhou University, Zhengzhou, Henan 450001, P.R. China; \\ ${ }^{6}$ College of Pharmacy, Keimyung University, Dalseo-gu, Daegu 704-701, Republic of Korea
}

Received November 21,2015; Accepted December 27, 2015

DOI: $10.3892 / \mathrm{ijo} .2016 .3365$

\begin{abstract}
Licochalcone B (Lico B), which belongs to the retrochalcone family, is isolated from the roots of Chinese licorice. Lico B has been reported to have several other useful pharmacological properties, such as anti-inflammatory, antibacterial,
\end{abstract}

Correspondence to: Professor Jung-Hyun Shim, Department of Pharmacy, College of Pharmacy, Mokpo National University, 1666 Youngsan-ro, Muan-gun, Jeonnam 534-729, Republic of Korea E-mail: s1004jh@gmail.com

Professor Jung-Il Chae, Department of Dental Pharmacology, School of Dentistry and Institute of Oral Bioscience, Chonbuk National University, 567 Baekje-daero, Deokjin-gu, Jeonju-si, Jeollabuk-do 561-756, Republic of Korea

E-mail: jichae@jbnu.ac.kr

${ }^{*}$ Contributed equally

Abbreviations: OSCC, oral squamous cell carcinoma; Lico, licochalcone; ROS, reactive oxygen species; DR, death receptor; MMP, mitochondrial membrane potential; Apaf-1, apoptotic protease activating factor-1; C-PARP, cleaved poly (ADP-ribose) polymerase; TRAIL, tumor necrosis factor-related apoptosisinducing ligand; FADD, Fas-associated death domain; ER, endoplasmic reticulum; CHOP, CCAAT/enhancer-binding protein homologous protein; DMEM, Dulbecco's modified Eagle's medium; FBS, fetal bovine serum; P/S, penicillin and streptomycin; PBS, phosphate buffered saline; Mcl-1, myeloid cell leukemia-1; DAPI, 4'-6-diamidino-2-phenylindole; RT, room temperature; 7-AAD, 7-aminoactinomycin D; PS, phosphatidylserine; MMP-9, matrix metalloproteinases-9; IAP, inhibitor of apoptosis; PI, propidium iodide

Key words: Licochalcone $\mathrm{B}$, oral squamous cell carcinoma, endoplasmic reticulum stress-mediated cell death, death receptor, reactive oxygen species, $\mathrm{CHOP}$, mitochondrial membrane potential, apoptosis antioxidant, antiulcer, anticancer, and anti-metastasis activities. We elucidated the underlying mechanism by which Lico B can induce apoptosis in oral squamous cell carcinoma (OSCC). Our results showed that exposure of OSCC cells (HN22 and HSC4) to Lico B significantly inhibited cell proliferation in a timeand concentration-dependent manner. Lico B caused cell cycle arrest at G1 phase along with downregulation of cyclin D1 and upregulation of p21 and p27 proteins. Lico B also facilitated the diffusion of phospholipid phosphatidylserine (PS) from inner to outer leaflets of the plasma membrane with chromatin condensation, DNA fragmentation, accumulated sub-G1 population in a concentration-dependent manner. Moreover, Lico B promoted the generation of reactive oxygen species (ROS), which, in turn, can induce CHOP, death receptor (DR) 4 and DR5. Lico B treatment induced downregulation of anti-apoptotic proteins (Bid and $\mathrm{Bcl}_{\mathrm{xl}}$ and $\mathrm{Mcl}-1$ ), and upregulation of pro-apoptotic protein (Bax). Lico B also led to the loss of mitochondrial membrane potential (MMP), resulting in cytochrome c release. As can be expected from the above results, the apoptotic protease activating factor-1 (Apaf-1) and survivin were oppositely expressed in favor of apoptotic cell death. This notion was supported by the fact that Lico B activated multi-caspases with cleavage of poly (ADP-ribose) polymerase (PARP) protein. Therefore, it is suggested that Lico B is a promising drug for the treatment of human oral cancer via the induction of apoptotic cell death.

\section{Introduction}

Oral squamous cell carcinoma (OSCC), accounting for over $90 \%$ of head and neck cancers, is the most common malignancy of oral cavity cancers, with more than half a million new patients diagnosed annually, and metastasis to lymph node frequently takes place (1-3). Since most patients are diagnosed with OSCC after the cancer has progressed to an advanced stage, the 5-year survival rate remains low $(4,5)$. In spite of great advances of early diagnosis and cancer treatment, the 5 -year survival rate for patients with OSCC remains $<50 \%$ and has not improved over the last few decades (3). Consequently, 
there is a need for development of effective therapeutic agents that could prevent and treat OSCC.

Herbal medicine has been regarded as an alternative approach to modern medicine and there have been various efforts to find active components with better anticancer potency and less side effects (6). Licorice, the root and rhizome of several Glycyrrhiza species (Leguminosase) (7), has been used as a flavouring agent, and traditional medicine for gastric ulcer, bronchial asthma and inflammation (8). There have been many studies on the biological effects of active ingredients of licorice, such as anti-inflammatory, anti-microbial, antioxidative, antiulcer, cytoprotective and cytotoxic activities (9). Licochalcone B (Lico B), a chalconoid presents in the root of Glycyrrhiza species, not only inhibit cell proliferation, but also to induce cell cycle arrest and apoptosis (10). Possibly, Lico B could be a promising alternative compound to traditional anticancer agents.

Apoptosis (programmed cell death), triggers an extrinsic (death receptor-mediated signaling) or an intrinsic (mitochondria-mediated signaling) pathway to activate caspase-3, leading to morphological alterations (e.g., DNA fragmentation, cell shrinkage, membrane blebbing and chromatin condensation) $(11,12)$. Tumor necrosis factor-related apoptosis-inducing ligand (TRAIL), a member of the TNF cytokine superfamily, interacts with five different receptors to coordinate cancer cell apoptosis. Among these receptors, only death receptor (DR)4 and DR5 have cytoplasmic death domains and are able to transduce the apoptotic signal of TRAIL by association with the Fas-associated death domain (FADD) protein, transmitting the apoptotic death signals into the cancer cells (13). Activation of caspase- 8 causes cleavage of $\mathrm{Bid}$ and $\mathrm{Bcl}-2$ family proteins and induces releasing of cytochrome $\mathrm{c}$ from mitochondria to cytosol. The mitochondria-mediated pathway is regulated by Bcl-2 family proteins such as Bax and Bak. Apoptotic induction is accompanied by the release of cytochrome $\mathrm{c}$ from mitochondria, which mediate apoptosis in both the extrinsic and intrinsic pathways. The resulting cytochrome $\mathrm{c}$ forms the apoptosome with apoptotic protease activating factor-1 (Apaf1), ATP and procaspase-9 (14). Thus, many studies have shown that the induced expression of DR4 and DR5 contributes to apoptosis caused by cancer drugs (13). Moreover, reactive oxygen species (ROS) induce endoplasmic reticulum (ER) stress-induced apoptosis via modulation of CCAAT/enhancerbinding protein homologous protein (CHOP) $(14,15)$. That is, overexpression of CHOP promotes apoptosis through downregulation of cell survival proteins and upregulation of DR4 and DR5 $(16,17)$.

However, mechanism of Lico B-induced apoptosis in OSCC have not been well defined. This study suggests that Lico B inhibits cell growth and induces apoptosis in OSCC HN22 and HSC4 cells.

\section{Materials and methods}

Reagents and antibodies. We prepared Lico B with 95\% purity according to the method previously reported (18). Dulbecco's modified Eagle's medium (DMEM), fetal bovine serum (FBS), trypsin, penicillin and streptomycin $(\mathrm{P} / \mathrm{S})$ and phosphatebuffered saline (PBS) were purchased from Thermo Scientific (Logan, UT, USA). Antibodies against cyclin D1, p21, p27, actin, CHOP, DR4, DR5, cytochrome c, $\alpha$-tubulin, MTCO1, Apaf-1, survivin, Bid, Bax, Bcl- ${ }_{x 1}$, myeloid cell leukemia-1 (Mcl-1) and poly (ADP-ribose) polymerase (PARP) were from Santa Cruz Biotechnology, Inc. (Santa Cruz, CA, USA). A 4',6-diamidino-2-phenylindole (DAPI) was obtained from Sigma-Aldrich, Inc. (St. Louis, MO, USA).

Cell culture. HN22 and HSC4 cells are human oral squamous cancer cell lines. HN22 cells were provided by Dankook University (Cheonan, Korea) and HSC4 cells were provided by Hokkaido University (Hokkaido, Japan) (19). Both cell types were maintained in DMEM supplemented with $10 \%$ FBS and $100 \mathrm{U} / \mathrm{ml}$ each of $\mathrm{P} / \mathrm{S}$ at $37^{\circ} \mathrm{C}$ in a $5 \%$ $\mathrm{CO}_{2}$ incubator.

Trypan blue staining. HN22 (6x10 $/ 6$-well plate) and HSC4 ( $7.5 \times 10^{4} / 6$-well plate) cells were grown for $24 \mathrm{~h}$, and then treated with various concentrations of Lico B $(0,10,20$ and $30 \mu \mathrm{M})$. After treatment with Lico B for 24 and $48 \mathrm{~h}$, cells were trypsinized and resuspended in complete medium. Each sample was mixed with trypan blue solution. Colored (nonviable) and dye-excluding (viable) cells were counted on a haemocytometer.

Annexin $V$ staining. HN22 (6x $10^{4} / 6$-well plate) and HSC4 (7.5x10 $/ 6$-well plate) cells were seeded and allowed to incubate overnight in 10\% FBS-containing DMEM. At $48 \mathrm{~h}$ after treatment with different concentration of Lico B $(0,10,20$ and $30 \mu \mathrm{M})$, cells were harvested by trypsinization and analyzed for the detection of early/late apoptosis and cell death using Annexin V and Dead Cell kit (EMD Millipore Corp., Billerica, MA, USA) according to the manufacturer's instructions. The percentage of apoptotic cells was calculated from triplicate samples by statistical analysis of dot plot using Muse Cell Analyzer (Merck Millipore).

DAPI staining. After treatment with Lico B, the HN22 and HSC4 cells were harvested by trypsin digestion. The cells were washed in ice-cold PBS and fixed with $100 \%$ methanol at room temperature (RT) for $1 \mathrm{~h}$. Fixed cells were stained with DAPI solution $(2 \mu \mathrm{g} / \mathrm{ml})$ and deposited on slides at RT in the dark. DAPI-stained cells were observed through a FluoView confocal laser microscope (Flouview FV10i, Olympus Corp., Tokyo, Japan).

Cell cycle. HN22 (6x10 $/ 6$-well plate) and HSC4 (7.5x 10 $/ 6$-well plate) cells were seeded and exposed to Lico B (0, 10, 20 and $30 \mu \mathrm{M}$ ) for $48 \mathrm{~h}$. The harvested cells were washed in PBS and $200 \mu \mathrm{l}$ of Muse cell cycle reagent (EMD Millipore Corp.) was added. The cells were incubated for $30 \mathrm{~min}$ at RT in the dark. Cell cycle distribution was analyzed by Muse Cell Analyzer (Merck Millipore).

Western blotting. The cell lysates were prepared using PRO-PREP ${ }^{\mathrm{TM}}$ Protein Extraction Solution (iNtRON Biotechnology, Korea) and then supernatants were collected by centrifugation. Samples containing equal amounts of protein were separated by SDS-PAGE and transferred to polyvinylidene difluoride membrane. The membranes were blocked with 5\% skim milk in PBS with $0.1 \%$ Tween-20 at 
A

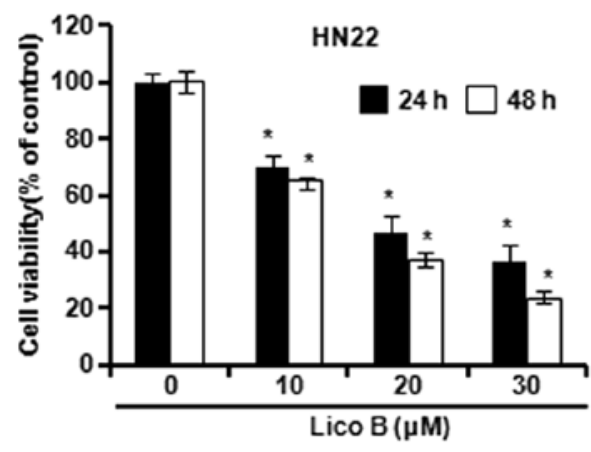

B

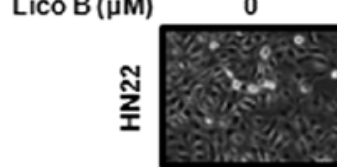

Lico B ( $\mu \mathrm{M})$

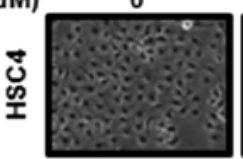

C

HN22

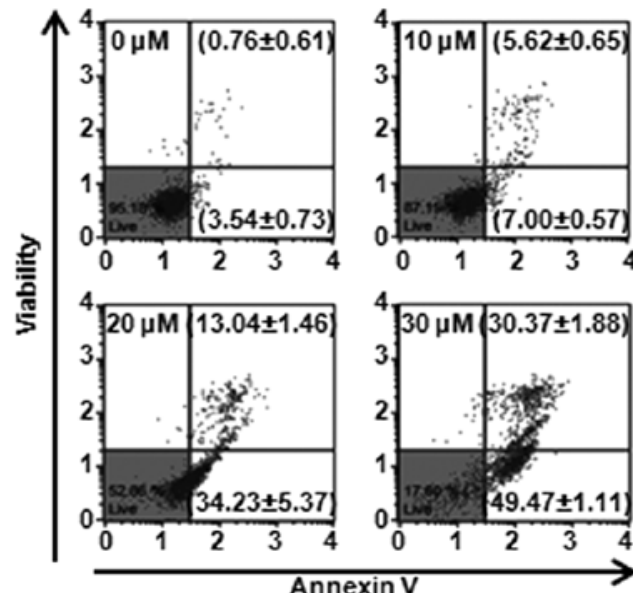

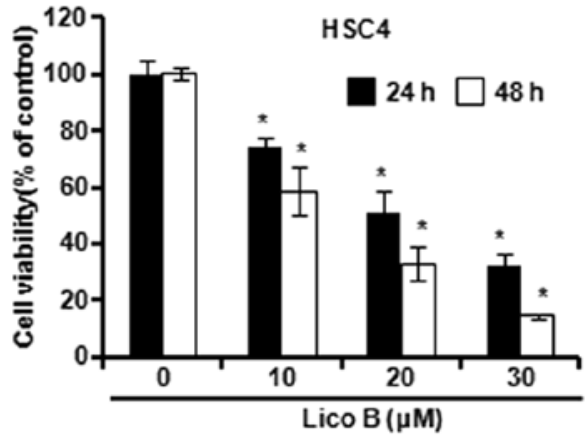

30
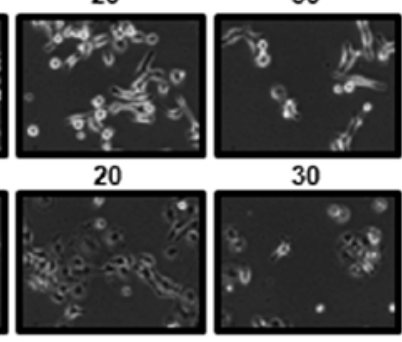

HSC 4

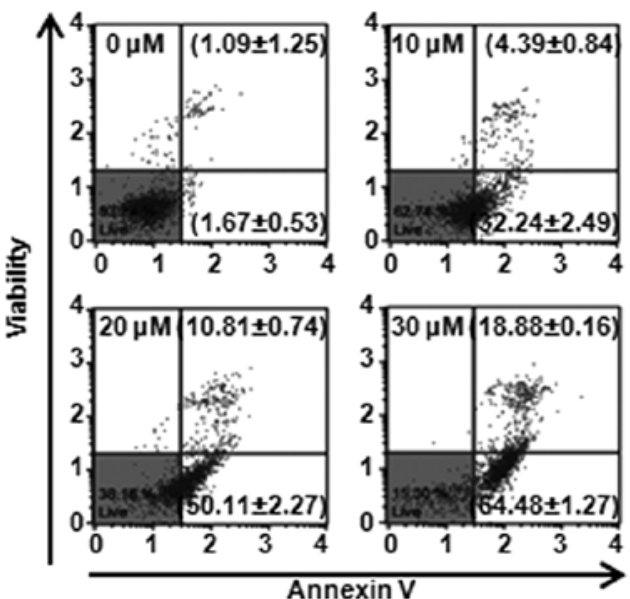

Figure 1. The effects of Lico B on cell viability and apoptosis in HN22 and HSC4 cells. (A) HN22 and HSC4 Cells were treated with various concentration of Lico B $(0,10,20$ and $30 \mu \mathrm{M})$ for 24 and $48 \mathrm{~h}$. Cell viability was assessed by Trypan blue staining. The graph is representative of three independent experiments and shown as means \pm SD. ${ }^{*} p<0.05$ compared with respective controls. (B) Morphological changes were observed using an optical microscope. (C) Flow cytometric analysis was carried out after Annexin V/7-AAD double staining. Dot plots were divided in quadrants according to fluorescence intensity: live (lower left), dead (upper left), early apoptotic (lower right) and late apoptotic (upper right).

RT. Membranes were probed with primary antibodies at $4^{\circ} \mathrm{C}$ overnight with mild shaking and then washed with PBST for a total of $30 \mathrm{~min}$. Finally, membranes were incubated with the secondary antibodies for $2 \mathrm{~h}$ at RT. Antibody-bound protein bands were visualized using ECL Plus Western Blotting Detection system (Santa Cruz Biotechnology, Inc.).

Measurement of intracellular ROS. Muse Oxidative Stress kit (EMD Millipore Corp.) was used to examine the effect of Lico B on the generation of ROS. Briefly, cells from each treatment were harvested, washed with PBS, and then resuspended in Muse Oxidative Stress Reagent working solution for $30 \mathrm{~min}$ at $37^{\circ} \mathrm{C}$ in the dark. Intracellular reactive oxygen species was measured by flow cytometry using Muse Cell Analyzer (Merck Millipore).

Mitochondrial membrane potential (MMP) detection assay. MMP was assessed using Muse MitoPotential kit (EMD
Millipore Corp.) according to the manufacturer's protocol. Briefly, HN22 and HSC4 cells treated with Lico B were harvested to observe quantitatively MMP. The cells were incubated with Muse MitoPotential working solution at $37^{\circ} \mathrm{C}$ for $20 \mathrm{~min}$. Then, $5 \mu \mathrm{l}$ of 7-aminoactinomycin D (7-AAD) was added and samples were further incubated at RT for $5 \mathrm{~min}$. Muse Cell Analyzer (Merk Millipore) was employed to detect MMP.

Multi-caspase assay. The activity of caspase-1, -3, -4, -5, -6, $-7,-8$ and -9 was measured using a Muse Multi-caspase kit (MCH100109, Merck Millipore) according to the manufacturer's instructions. HN22 and HSC4 cells were incubated with various concentrations of Lico $\mathrm{B}(0,10,20$ and $30 \mu \mathrm{M})$ for $48 \mathrm{~h}$. Cells were harvested and incubated with $5 \mu \mathrm{l}$ of Muse Multi-Caspase reagent working solution at $37^{\circ} \mathrm{C}$ for $30 \mathrm{~min}$. Then, $150 \mu \mathrm{l}$ of Muse Caspase 7-AAD working solution was 

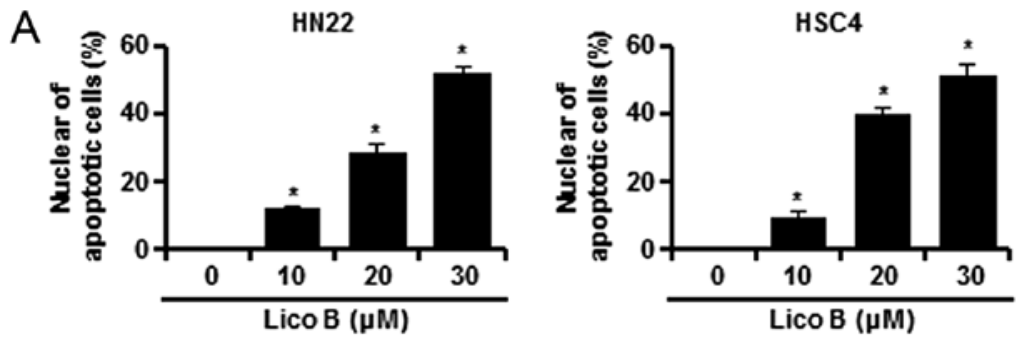

B

HN22
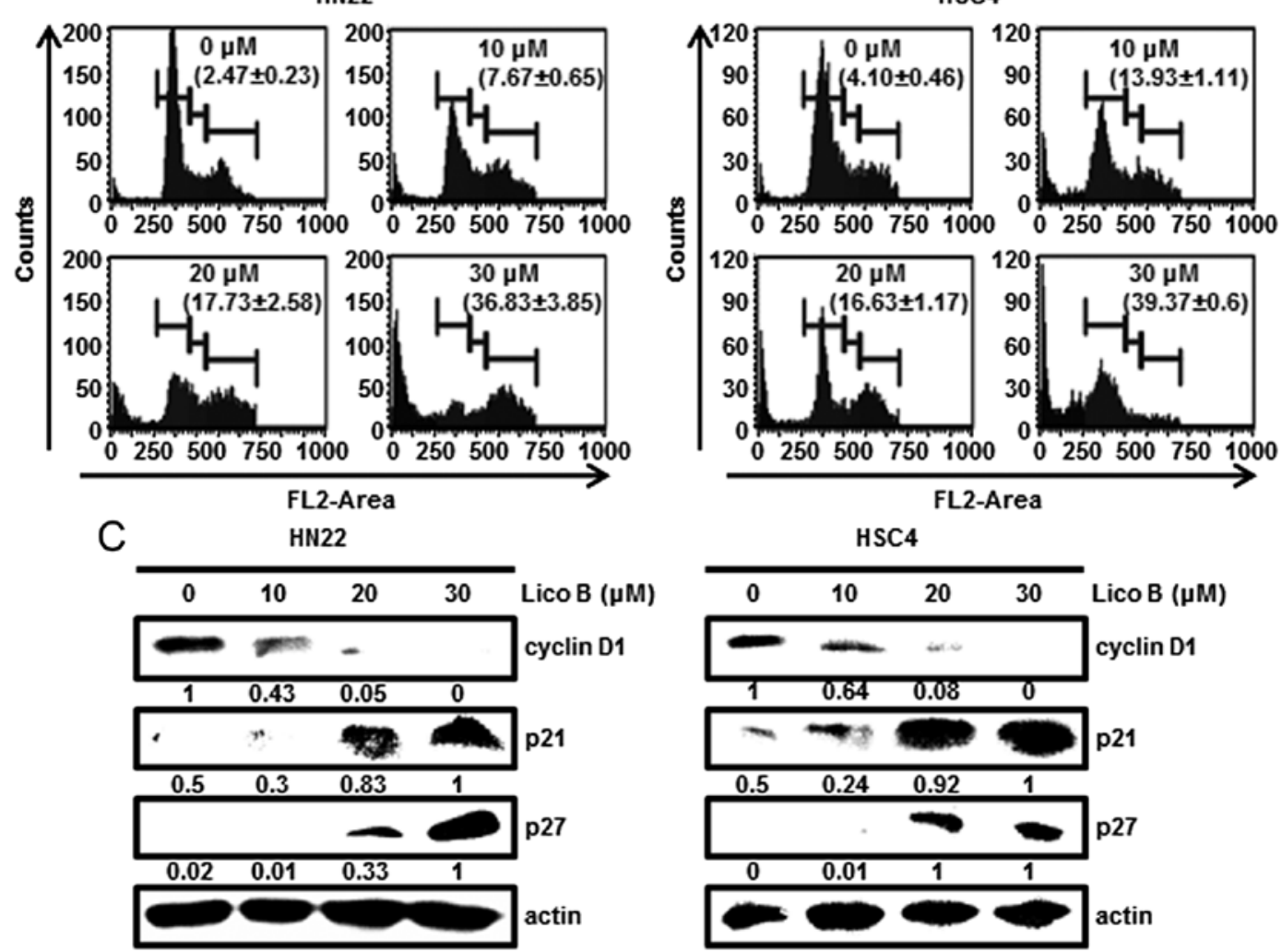

HSC4

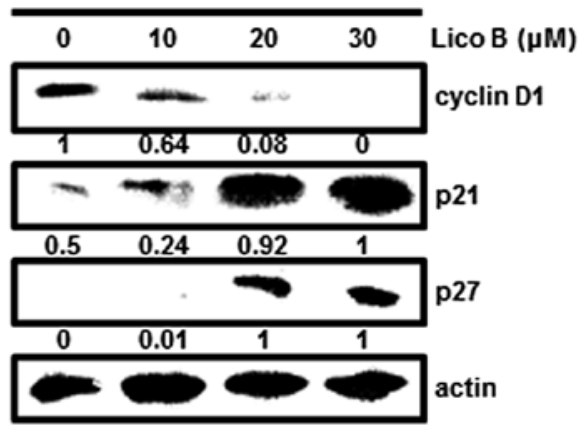

Figure 2. The effect of Lico B on the DNA condensation, fragmentation and the cell cycle. (A) Nuclear features of the cells assessed by DAPI staining. DAPI-stained cells were quantified, apoptotic cell numbers are indicated in the graph as means \pm SD of triplicate experiments. "p $<0.05$ compared to control group. HN22 and HSC4 cells were treated with different concentration $(0,10,20$ and $30 \mu \mathrm{M})$ of Lico B for $48 \mathrm{~h}$. (B) The cell cycle distribution was analyzed by Muse Cell Analyzer after PI staining. The data indicate sub- $\mathrm{G}_{1}$ population and the means \pm SD of three different experiments. (C) Total cell lysates were prepared and assessed by western blotting with specific antibodies. Cyclin D1, p21, p27 and actin-specific bands were quantified by ImageJ software, then cyclin D1, p21 and p27 expression levels were normalized to actin.

added to each sample. Multi-caspase assay was performed with Muse Cell Analyzer (Merck Millipore).

Statistical analysis. Using Student's t-test, the statistical significance was assessed. The result with $\mathrm{p}$-value $<0.05$, indicates statistical significance of data.

\section{Results}

Inhibition of cell proliferation by Lico B. Trypan blue staining was performed to determine the viability of HN22 and HSC4 cells treated with Lico B $(0,10,20$ and $30 \mu \mathrm{M})$ for 24 and $48 \mathrm{~h}$, and then live cells were counted using a haemocytometer. As shown in Fig. 1A, cell viability was significantly decreased in a concentration- and time-dependent manner by Lico B (Fig. 1A). The $\mathrm{IC}_{50}$ value was $15 \mu \mathrm{M}$ for $\mathrm{HN} 22$ cells and $13 \mu \mathrm{M}$ for HSC4 cells at $48 \mathrm{~h}$. Cell morphology was observed under an inverted phase contrast microscope after the cells were treated with Lico B for $48 \mathrm{~h}$, and images were obtained (Fig. 1B). Significant cell shrinkage, formation of plasma membrane blebs and a decreased cellular attachment rate were observed in the Lico B-treated groups. These results suggest that Lico B has an inhibitory effect on the cell growth of HN22 and HSC4 cells.

Lico B induces apoptosis in HN22 and HSC4 cells. Phosphatidylserine (PS) of normal cells are mainly distributed on the inner layer of the membrane. However, early apoptotic cells transpose PS on the outer layer of the membrane (20). Annexin V can detect PS on the outer membrane, designating early apoptotic cells while 7-AAD can permeate into plasma membrane and bind to DNA when the membrane of cells lose integrity. Annexin V and 7-AAD absorb laser with different wavelengths so that both fluorophores can be analyzed at 

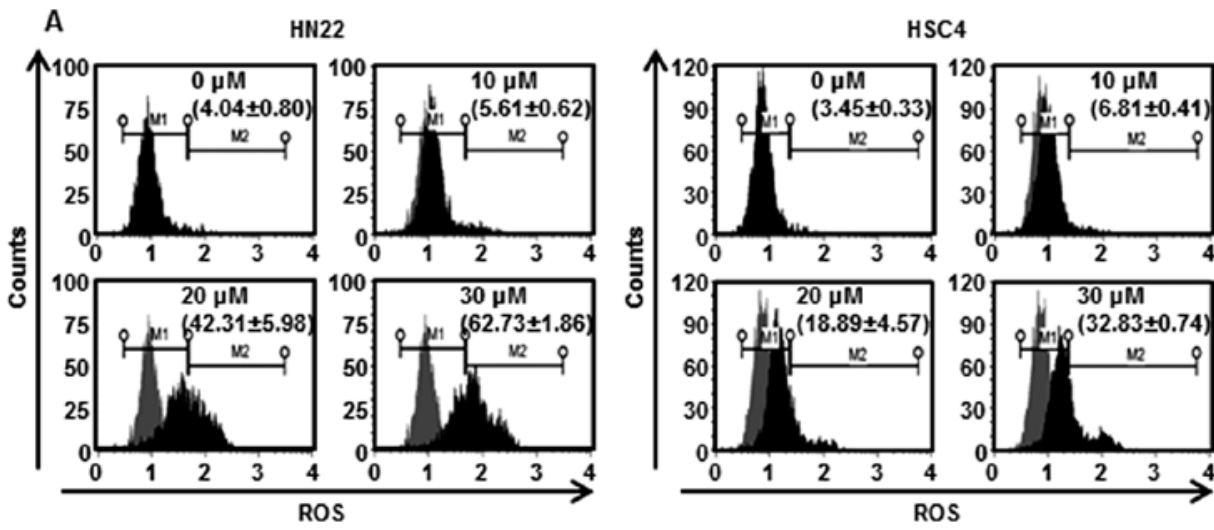

B

HN22
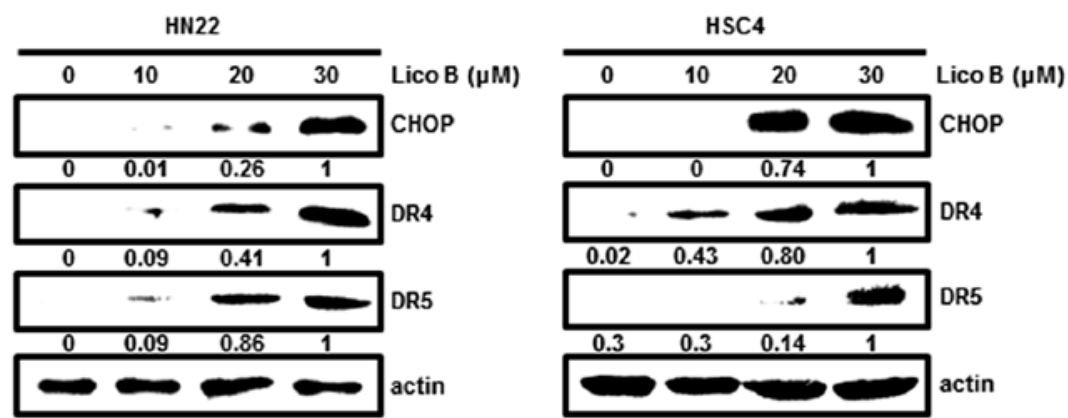

C

HN22
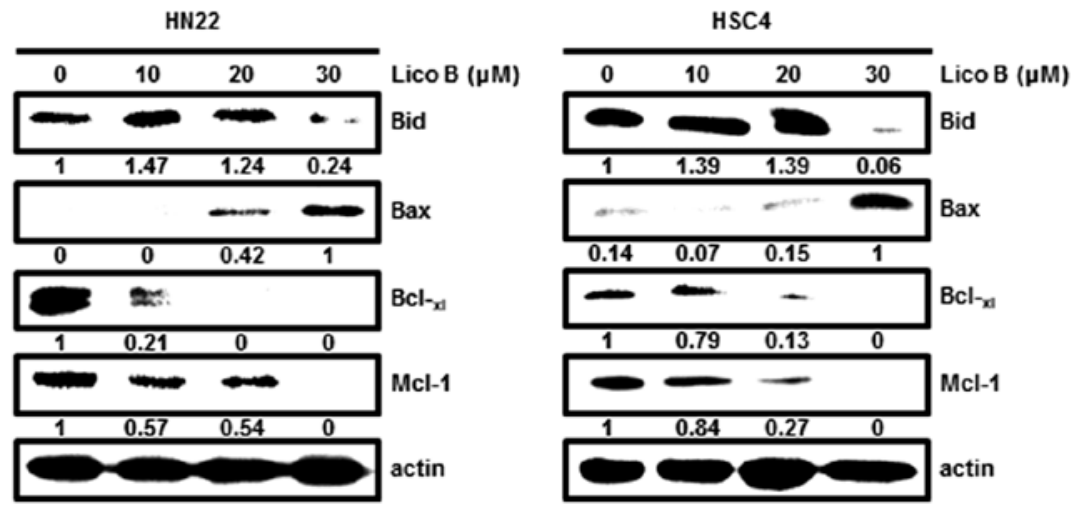

Figure 3. The effect of Lico B on the expression of apoptosis regulatory proteins of HN22 and HSC4 cells. HN22 and HSC4 cells were treated with 0 , 10 , 20 and $30 \mu \mathrm{M}$ Lico B for $48 \mathrm{~h}$. (A) The cells were incubated at $37^{\circ} \mathrm{C}$ in the dark for $30 \mathrm{~min}$ with Muse Oxidative Stress Reagent working solution. Reactive oxygen species generation was measured using a Muse Cell Analyzer. CHOP, DR4, DR5 (B) and Bcl-2 family proteins (C) were determined by western blotting using specific antibodies. The values were determined by ImageJ software.

the same time (20). To determine whether the Lico B could induce apoptosis in HN22 and HSC4 cells, we stained cells with Annexin V/7-AAD for analysis by flow cytometry. As shown in Fig. 1C, Lico B caused apoptosis of OSCC cells in a concentration-dependent manner. The apoptotic cells of HN22 were $12.62 \pm 1.22,47.27 \pm 6.83,79.84 \pm 2.99 \%$ at 10,20 and $30 \mu \mathrm{M}$ of Lico B, respectively (Fig. 1C, left). In HSC4, population of apoptotic cells was $36.63 \pm 3.33$, 60.92 \pm 3.01 , $83.36 \pm 1.43 \%$ at 10,20 and $30 \mu \mathrm{M}$ of Lico $\mathrm{B}$, respectively (Fig. 1C, right). Additionally, the characteristic appearance of apoptosis was analyzed by DAPI staining. HN22 and HSC4 cells treated with Lico B presented nuclear shrinkage, chromatin condensation and fragmentation of nuclear bodies compared to control (Fig. 2A). The cell cycle distribution was assessed after PI staining by Muse Cell Analyzer. As shown in Fig. 2B, the treatment of cells with Lico B at $30 \mu \mathrm{M}$ caused $36.83 \pm 3.85$ or $39.37 \pm 0.6 \%$ induction of sub- $G_{1}$ phase in $H N 22$ (Fig. 2B, left) and HSC4 (Fig. 2B, right), respectively. Overall, these findings suggest that Lico B can induce apoptosis in HN22 and HSC4 cells.

Lico B induces $G_{I}$ phase arrest in HN22 and HSC4 cells. To determine the effect of Lico B on the cell cycle progression, HN22 and HSC4 cells were treated with different concentrations of Lico B $(0,10,20$ and $30 \mu \mathrm{M})$ for $48 \mathrm{~h}$ and the cell cycle distribution was assessed by Muse Cell Analyzer. Fig. 2B showed that HN22 and HSC4 cells accumulated in $\mathrm{G}_{1}$ phase after treatment of Lico B (Fig. 2B). To confirm $\mathrm{G}_{1}$ block in cell cycle progression, the level of $\mathrm{G}_{1}$ cell cycle components was monitored by western blot analysis. Fig. 2C showed that Lico B increased the protein levels of p21 and p27, and decreased the levels of cyclin D1 (Fig. 2C). These results support that Lico B causes $\mathrm{G}_{1}$ phase arrest in OSCC cells.

Lico $B$ induces endoplasmic reticulum (ER) stress and expression of death receptor (DR) 4, DR5 in HN22 and HSC4 cells. 

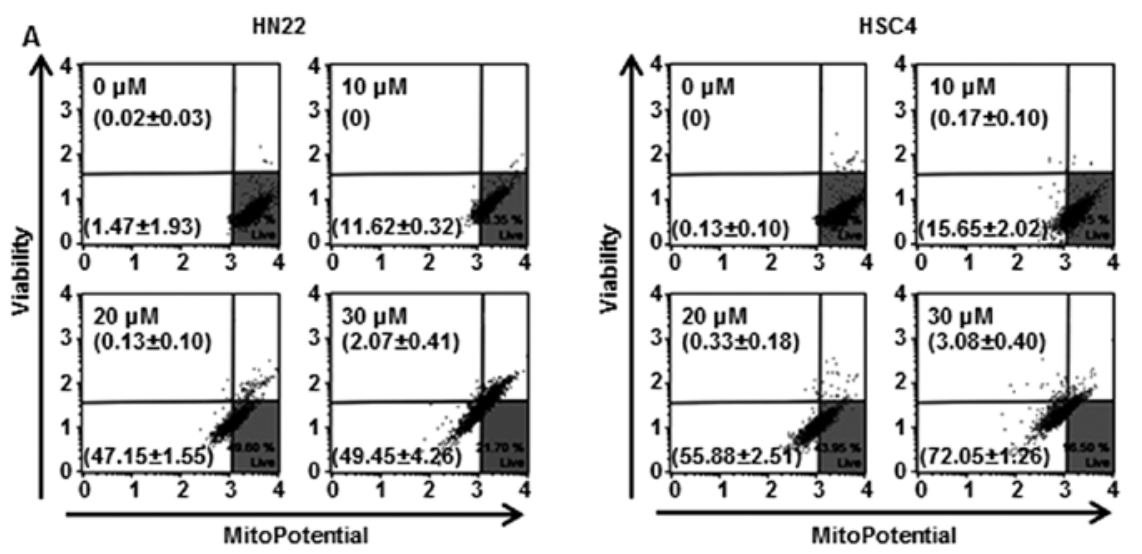

B

HN22
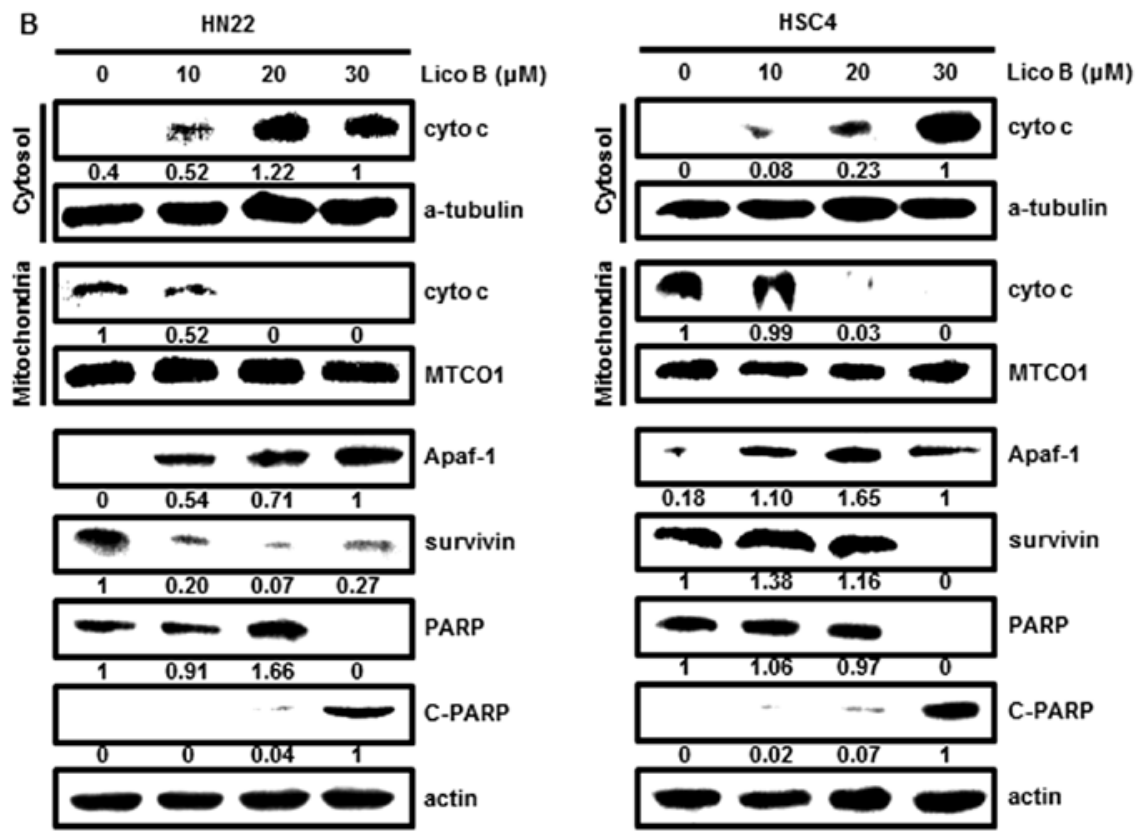

Figure 4. The effect of Lico B on mitochondrial membrane potential and western blot analysis of cytochrome c (cyto c) in cytosolic and mitochondrial fractions from OSCC cells. HN22 and HSC4 cells were untreated or incubated in media containing 10, 20 and $30 \mu \mathrm{M}$ Lico B for $48 \mathrm{~h}$. (A) Mitochondrial membrane potential was measured with Muse Cell Analyzer by flow cytometry. The shift-down fluorescence from right to left indicates depolarization of mitochondrial membrane potential. The data are expressed as the means \pm SD of three experiments of triplicates. (B) Cyto $c$ of cytosolic fraction and mitochondrial fraction were assessed by western blotting. Also, Apaf-1, survivin, poly (ADP-ribose) polymerase (PARP) and cleaved PARP (C-PARP) expression of total cell lysates were analyzed by western blotting. Relative expression of cyto c, Apaf-1, survivin, PARP and C-PARP was quantified by ImageJ Instrument software after normalization with the density of interval control ( $\alpha$-tubulin, MTCO1 and actin).

Overwhelming ER stress resulted from accumulation of ROS has been known to trigger apoptotic signaling $(15,21)$. As shown in Fig. 3A, ROS production from HN22 and HSC4 cells treated with Lico B was significantly increased. CHOP is one of the components of ER stress-mediated apoptosis pathway (16). To assess the effect of Lico B on CHOP expression in HN22 and HSC4 cells, western blotting was performed. Fig. 3B showed that the protein levels of CHOP were increased in a dose-dependent manner by Lico B. CHOP is suggested to induce apoptosis through upregulation of DR4 and DR5 expression (16). We also observed that Lico B treatment increased dose-dependently DR4 and DR5 in HN22 and HSC4 cells (Fig. 3B). These results showed that treatment of OSCC cells with Lico B induced ER stress, which, in turn, upregulates CHOP, DR4 and DR5.

Lico $B$ modulates the level of proteins related to apoptosis in HN22 and HSC4 cells. Dynamic changes in Bcl-2 family proteins were associated with death receptor-mediated apoptosis (22). Bcl- $_{\mathrm{xl}}$ functions as an anti-apoptotic protein, whereas Bax is pro-apoptotic protein (23). Bid is then truncated to induce apoptosis. Downregulation of Mcl-1 protein may be required to initiate a cascade of apoptotic signals leading to release of cytochrome c (5). To investigate whether expression of apoptosis regulatory proteins may be modulated by Lico B, the protein levels of Bid, Bax, Bcl- ${ }_{x 1}$ and $\mathrm{Mcl}-1$ in $\mathrm{HN} 22$ and HSC 4 cells treated with Lico B were analyzed by western blotting. Fig. 3C showed that the levels of pro-apoptotic Bax were increased and the expression levels of $\mathrm{Bid}, \mathrm{Bcl}_{-\mathrm{xl}}$ and $\mathrm{Mcl}-1$ were decreased in a concentration-dependent manner.

Lico B induces mitochondrial membrane depolarization and mitochondria-mediated apoptosis in OSCC cells. Death receptor induces apoptosis through the disruption of the mitochondrial membrane potential (MMP) and sequential release of cytochrome c from mitochondria into the cytosol (24). The 
HN22

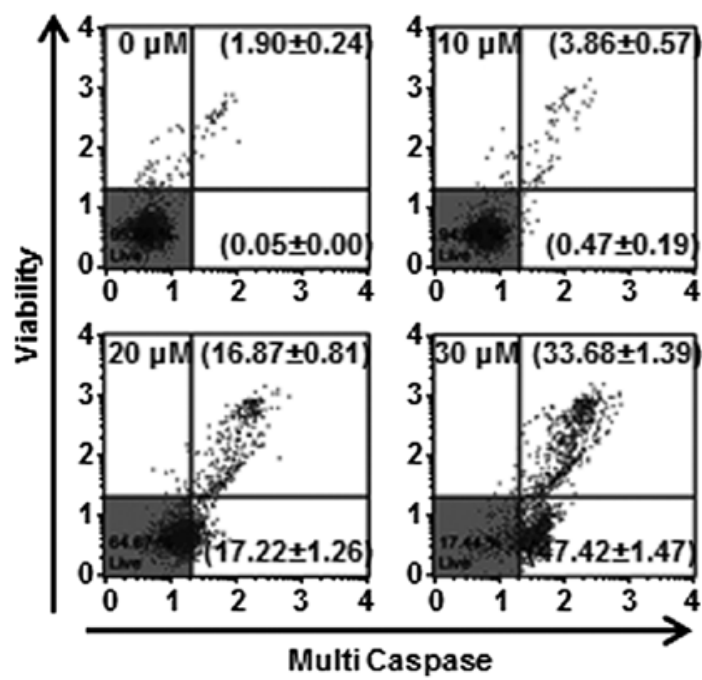

HSC4

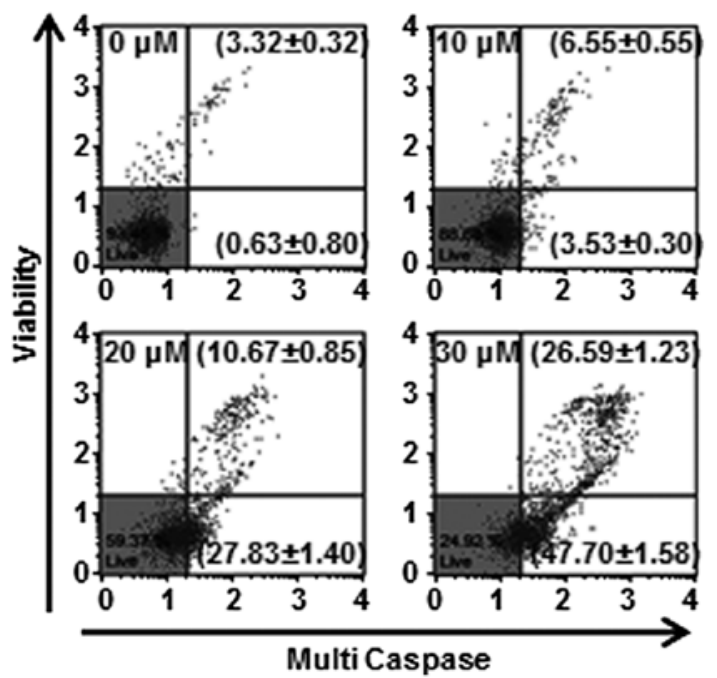

Figure 5. The effect of Lico B on activity of caspases. HN22 and HSC4 cells were exposed to Lico B (0, 10, 20 and $30 \mu \mathrm{M})$ for 48 h. Activity of caspases was evaluated by Muse Cell Analyzer. Fluorescence intensity for multi-caspase is indicated on the x-asis, and 7-AAD is indicated on the y-axis. The population of stained/unstained cells is indicated in each quadrant. Results represent the means \pm SD from triplicates.

MMP was measured by Muse Cell Analyzer with cationic, lipophilic dye. The dye permeates mitochondrial membrane of intact cells and accumulates within inner membrane. Thus, cells with depolarized mitochondria show a decrease in fluorescence (25). The data indicated that treatment of cells with Lico B induced a loss of MMP in HN22 and HSC4 cells (Fig. 4A). The loss of MMP leads to the release of cytochrome $\mathrm{c}$ into the cytosol then, triggering the downstream processes in the apoptotic cascade (26). To examine whether Lico B could cause the release of cytochrome $\mathrm{c}$ into the cytosol, subcellular distribution of cytochrome $\mathrm{c}$ in cytosol and mitochondria fraction were analyzed by western blotting. The results showed that cytochrome $\mathrm{c}$ in cytosol fraction of HN22 and HSC4 cells treated with Lico B $(0,10,20$ and $30 \mu \mathrm{M})$ was concentrationdependently increased (Fig. 4B). These results indicated that Lico B facilitated mitochondrial membrane depolarization and release of cytochrome $\mathrm{c}$ into the cytosol. Additionally, to investigate downstream target proteins including Apaf-1, survivin and PARP in mitochondria-mediated apoptosis, their expression was analyzed by western blotting with specific antibodies. As shown in Fig. 4B, the expression levels of survivin and PARP were diminished, but Apaf-1 and C-PARP were increased in a concentration-dependent manner by Lico B.

Lico B induces caspase-mediated apoptosis in HN22 and HSC4 cells. To examine whether Lico B-induced apoptosis would be associated with the activation of caspases, the expression levels and activity of caspases such as caspase-1, $-3,-4,-5,-6,-7,-8$ and -9 in the Lico B-treated OSCC cells were assessed using Muse Cell Analyzer. As shown in Fig. 5, HN22 and HSC4 cells treated with Lico B exhibited enhanced multi-caspase activity in a concentration-dependent manner. The multi-caspase activity of HN22 cells (Fig. 5, left) was $4.33 \pm 0.76,34.09 \pm 2.07$ and $81.1 \pm 2.86 \%$, respectively, at 10 , 20 and $30 \mu \mathrm{M}$ of Lico B compared with control. In HSC4 cells (Fig. 5, right), multi-caspase activity was 10.08 \pm 0.85 ,
$38.5 \pm 2.25$ and $74.29 \pm 2.81 \%$ at 10,20 and $30 \mu \mathrm{M}$ of Lico B, respectively.

\section{Discussion}

Natural extracts derived from edible plants have been investigated as alternative products of cancer drugs based on safety and efficacy (27). Chalcones are ubiquitous natural products with a wide variety of reported biological activities such as antiviral, anti-microbial, anti-inflammatory and anti-cancer effect $(6,28)$. Recent studies have reported that Lico B, a chalcone extracted from licorice, inhibits cell proliferation and induces cell cycle arrest and apoptosis in tumor cells (6). Lico B has been reported to attenuate bladder cancer cell migration, adhesion and invasion by downregulating the protein expression and suppressing the activity of matrix metalloproteinase 9 (10).

Oral squamous cell carcinoma (OSCC) is the most common neoplasm of oral cavity cancers (4). Despite great advances in cancer diagnosis and therapy, there has been little improvement in the 5-year survival rate of oral cancer patients (27). Anticancer effects of the Lico B have been reported in a variety of cancers, but has not been examined previously in OSCC. To better understand the action mechanisms of Lico B on OSCC cell lines, we further checked the intracellular signaling pathway. We found that Lico B induced apoptosis by intrinsic and extrinsic apoptotic pathways in HN22 and HSC4 cells. We found that Lico B significantly not only inhibited cell growth in a concentration- and timedependent manner, but also induced apoptosis in OSCC cells (HN22 and HSC4).

The morphological alterations such as cell shrinkage, chromatin condensation and DNA fragmentation supported Lico B-induced apoptotic induction. p21 and p27 proteins are members of the Cip/Kip family, and regulate cell cycle progression (29). Cyclin D1 is a key protein at $G_{1}$ phase in the 
cell cycle progression. This protein dimerizes with CDK4/6 to regulate the $\mathrm{G}_{1} / \mathrm{S}$ phase transition and enter the $\mathrm{S}$ phase. Thus, cyclin D1 is involved in the development and progression of various tumors (30). Our results showed that percentage of cells was increased in the sub- $\mathrm{G}_{1}$ phase during Lico B treatment, arresting in $\mathrm{G}_{1}$ phase. In OSCC cells, Lico B inhibits cell cycle progression as a result of reduced cyclin D1, and increased p21 and p27 expression. Deregulation of the cell cycle is frequent during cancer development (6). Therefore, inhibition of cell cycle progression is considered as useful approach to control of cancer cell growth.

In several studies, phytochemicals exhibited anticancer activities by the activation of death receptor and loss of MMP in tumor cells (31), and anticancer agents can enhance ROS production associated with apoptosis in cells (32). ER stress-induced response mediates the expression of the apoptosis-relevant gene such as CHOP, DR4 and DR5 $(15,33)$. CHOP plays a decisive role in the expression of death receptor (17). Our results indicated that Lico B induced DR4 and DR5 expression through regulation of ROS-CHOP mediated pathway. The results suggest that Lico B induces apoptosis through the extrinsic pathway. The expression of Bcl-2 family proteins is directly linked to cell damage and there are important factors that make up the apoptosome (34). The mitochondrial function has been reported to be regulated by Bcl-2 family proteins such as anti-apoptotic (Bcl-2, $\mathrm{Bcl}_{\mathrm{x}}$ and $\mathrm{Mcl}-1$ ) and pro-apoptotic (Bax, Bad and Bak) proteins (35). Thus, changes in the levels of Bcl-2 family proteins can affect the mitochondrial physiology positively or negatively (36). The results showed that Lico B led to downregulation of anti-apoptotic Bid, $\mathrm{Bcl}_{-\mathrm{xl}}$ and $\mathrm{Mcl}-1$, and upregulation of proapoptotic Bax. Subsequently, Lico B resulted in a significant decrease in the level of MMP and release of cytochrome c from mitochondria to cytosol, finally executing cell death by activation of caspases (37).

The release of cytochrome $\mathrm{c}$ into the cytosol is regulated by the equilibrium between anti-apoptotic and pro-apoptotic proteins and plays an important role in the execution of apoptosis $(26,34)$. Additionally, Lico B treatment increased the expression of Apaf- 1 and cleavage of PARP, while it decreased inhibitor of apoptosis (IAP) family proteins (survivin) which are direct inhibitors of activated caspases (38). The activation of Bax and caspase is critical for initiation and amplification of apoptosis, and their activation is normally blocked by $\mathrm{Bcl}_{\mathrm{xl}}$ or IAP proteins (6). All these studies support that Lico B also induces apoptosis through the intrinsic pathway.

In summary, this study demonstrated that Lico B induced apoptosis in OSCC cells. Apoptosis was triggered through the extrinsic pathway by upregulating death receptor and also the intrinsic pathway by modulating $\mathrm{Bcl}-2$ and IAP family members. These findings provide the underlying molecular mechanisms for the anticancer effects of Lico B that can be useful in the treatment of human oral cancer.

\section{Acknowledgements}

This study was supported by Basic Science Research program through the National Research Foundation Korea (NRF). Funded by the Ministry of Education, Science and Technology (2014R1A1A2053500) and the Next-Generation
BioGreen 21 Program (PJ01116401) from Rural Development Administration, Republic of Korea.

\section{References}

1. Zeng G, Shen H, Yang Y, Cai X and Xun W: Licochalcone A as a potent antitumor agent suppresses growth of human oral cancer SCC-25 cells in vitro via caspase-3 dependent pathways. Tumour Biol 35: 6549-6555, 2014.

2. Neville BW and Day TA: Oral cancer and precancerous lesions. CA Cancer J Clin 52: 195-215, 2002.

3. Ni ZY, Lin FO, Liu DF and Xiao J: Decreased microRNA-143 expression and its tumor suppressive function in human oral squamous cell carcinoma. Genet Mol Res 14: 6943-6952, 2015.

4. Shin JA, Jung JY, Ryu MH, Safe S and Cho SD: Mithramycin A inhibits myeloid cell leukemia-1 to induce apoptosis in oral squamous cell carcinomas and tumor xenograft through activation of Bax and oligomerization. Mol Pharmacol 83: 33-41, 2013.

5. Shin JA, Kim JS, Kwon KH, Nam JS, Jung JY, Cho NP and Cho SD: Apoptotic effect of hot water extract of Sanguisorba officinalis L. in human oral cancer cells. Oncol Lett 4: 489-494, 2012.

6. Yuan X, Li T, Xiao E, Zhao H, Li Y, Fu S, Gan L, Wang Z, Zheng $\mathrm{Q}$ and Wang Z: Licochalcone B inhibits growth of bladder cancer cells by arresting cell cycle progression and inducing apoptosis. Food Chem Toxicol 65: 242-251, 2014.

7. Fu Y, Chen J, Li YJ, Zheng YF and Li P: Antioxidant and antiinflammatory activities of six flavonoids separated from licorice. Food Chem 141: 1063-1071, 2013.

8. Furusawa J, Funakoshi-Tago M, Mashino T, Tago K, Inoue H, Sonoda Y and Kasahara T: Glycyrrhiza inflata-derived chalcones, Licochalcone A, Licochalcone B and Licochalcone $\mathrm{D}$, inhibit phosphorylation of NF-kappaB p65 in LPS signaling pathway. Int Immunopharmacol 9: 499-507, 2009.

9. Park SY, Kim EJ, Choi HJ, Seon MR, Lim SS, Kang YH, Choi MS, Lee KW and Yoon Park JH: Anti-carcinogenic effects of non-polar components containing licochalcone $\mathrm{A}$ in roasted licorice root. Nutr Res Pract 8: 257-266, 2014.

10. Zhao H, Yuan X, Jiang J, Wang P, Sun X, Wang D and Zheng Q: Antimetastatic effects of licochalcone B on human bladder carcinoma T2 4 by inhibition of matrix metalloproteinases- 9 and NF- $\kappa$ B activity. Basic Clin Pharmacol Toxicol 115: 527-533, 2014.

11. Elmore S: Apoptosis: A review of programmed cell death. Toxicol Pathol 35: 495-516, 2007.

12. Lee MS, Cherla RP, Lentz EK, Leyva-Illades D and Tesh VL: Signaling through C/EBP homologous protein and death receptor 5 and calpain activation differentially regulate THP-1 cell maturation-dependent apoptosis induced by Shiga toxin type 1. Infect Immun 78: 3378-3391, 2010.

13. Zhu H, Liu XW, Ding WJ, Xu DQ, Zhao YC, Lu W, He QJ and Yang B: Up-regulation of death receptor 4 and 5 by celastrol enhances the anti-cancer activity of TRAIL/Apo-2L. Cancer Lett 297: 155-164, 2010.

14. Huang TT, Liu FG, Wei CF, Lu CC, Chen CC, Lin HC, Ojcius DM and Lai HC: Activation of multiple apoptotic pathways in human nasopharyngeal carcinoma cells by the prenylated isoflavone, osajin. PLoS One 6: e18308, 2011.

15. Farooqi AA, Li KT, Fayyaz S, Chang YT, Ismail M, Liaw CC, Yuan SS, Tang JY and Chang HW: Anticancer drugs for the modulation of endoplasmic reticulum stress and oxidative stress. Tumour Biol 36: 5743-5752, 2015.

16. Xu L, Su L and Liu X: PKCठ regulates death receptor 5 expression induced by PS-341 through ATF4-ATF3/CHOP axis in human lung cancer cells. Mol Cancer Ther 11: 2174-2182, 2012.

17. Prasad S, Yadav VR, Ravindran J and Aggarwal BB: ROS and CHOP are critical for dibenzylideneacetone to sensitize tumor cells to TRAIL through induction of death receptors and downregulation of cell survival proteins. Cancer Res 71: 538-549, 2011.

18. Wang Z, Liu Z, Cao Y, Paudel S, Yoon G and Cheon SH: Short and efficient synthesis of licochalcone $B$ and D through acidmediated Claisen-Schmidt condensation. Bull Korean Chem Soc 34: 3906-3908, 2013.

19. Cho JJ, Chae JI, Yoon G, Kim KH, Cho JH, Cho SS, Cho YS and Shim JH: Licochalcone A, a natural chalconoid isolated from Glycyrrhiza inflata root, induces apoptosis via Sp1 and Sp1 regulatory proteins in oral squamous cell carcinoma. Int J Oncol 45: 667-674, 2014. 
20. Janes SM, Ofstad TA, Campbell DH, Eddaoudi A, Warnes G, Davies D and Watt FM: PI3-kinase-dependent activation of apoptotic machinery occurs on commitment of epidermal keratinocytes to terminal differentiation. Cell Res 19: 328-339, 2009.

21. Fu HY, Okada K, Liao Y, Tsukamoto O, Isomura T, Asai M Sawada T, Okuda K, Asano Y, Sanada S, et al: Ablation of C/EBP homologous protein attenuates endoplasmic reticulum-mediated apoptosis and cardiac dysfunction induced by pressure overload. Circulation 122: 361-369, 2010.

22. Shin DY, Park YS, Yang K, Kim GY, Kim WJ, Han MH, Kang HS and Choi YH: Decitabine, a DNA methyltransferase inhibitor, induces apoptosis in human leukemia cells through intracellular reactive oxygen species generation. Int J Oncol 41: 910-918, 2012.

23. Habibie YS, Yokoyama S, Abdelhamed S, Awale S, Sakurai H, Hayakawa Y and Saiki I: Survivin suppression through STAT3 $/ \beta$-catenin is essential for resveratrol-induced melanoma apoptosis. Int J Oncol 45: 895-901, 2014.

24. Kim YS, Kim EA, Park KG, Lee SJ, Kim MS, Sohn HY and Lee TJ: Dioscin sensitizes cells to TRAIL-induced apoptosis through downregulation of c-FLIP and Bcl-2. Oncol Rep 28 : 1910-1916, 2012

25. Law CK, Kwok HH, Poon PY, Lau CC, Jiang ZH, Tai WC, Hsiao WW, Mak NK, Yue PY and Wong RN: Ginsenoside compound $\mathrm{K}$ induces apoptosis in nasopharyngeal carcinoma cells via activation of apoptosis-inducing factor. Chin Med 9: 11, 2014.

26. Hearps AC, Burrows J, Connor CE, Woods GM, Lowenthal RM and Ragg SJ: Mitochondrial cytochrome c release precedes transmembrane depolarisation and caspase-3 activation during ceramide-induced apoptosis of Jurkat T cells. Apoptosis 7: 387-394, 2002.

27. Choi ES, Cho SD, Jeon JG and Cho NP: The apoptotic effect of the hexane extract of Rheum undulatum L. in oral cancer cells through the down-regulation of specificity protein 1 and survivin. Lab Anim Res 27: 19-24, 2011.

28. Mahapatra DK, Bharti SK and Asati V: Chalcone scaffolds as anti-infective agents: Structural and molecular target perspectives. Eur J Med Chem 101: 496-524, 2015.
29. Yalcin A, Clem BF, Imbert-Fernandez Y, Ozcan SC, Peker S, O'Neal J, Klarer AC, Clem AL, Telang S and Chesney J: 6-Phosphofructo-2-kinase (PFKFB3) promotes cell cycle progression and suppresses apoptosis via Cdk1-mediated phosphorylation of p27. Cell Death Dis 5: e1337, 2014.

30. Alao JP: The regulation of cyclin D1 degradation: Roles in cancer development and the potential for therapeutic invention. Mol Cancer 6: 24, 2007.

31. Han MH, Lee WS, Jung JH, Jeong JH, Park C, Kim HJ, Kim G, Jung JM, Kwon TK, Kim GY, et al: Polyphenols isolated from Allium cepa L. induces apoptosis by suppressing IAP-1 through inhibiting PI3K/Akt signaling pathways in human leukemic cells. Food Chem Toxicol 62: 382-389, 2013.

32. Federico A, Cardaioli E, Da Pozzo P, Formichi P, Gallus GN and Radi E: Mitochondria, oxidative stress and neurodegeneration. J Neurol Sci 322: 254-262, 2012.

33. Kim IY, Kang YJ, Yoon MJ, Kim EH, Kim SU, Kwon TK, Kim IA and Choi KS: Amiodarone sensitizes human glioma cells but not astrocytes to TRAIL-induced apoptosis via CHOPmediated DR5 upregulation. Neuro Oncol 13: 267-279, 2011.

34. Kim CD, Cha JD, Li S and Cha IH: The mechanism of acacetininduced apoptosis on oral squamous cell carcinoma. Arch Oral Biol 60: 1283-1298, 2015.

35. Giam M, Huang DC and Bouillet P: BH3-only proteins and their roles in programmed cell death. Oncogene 27 (Suppl 1): S128-S136, 2008.

36. Ola MS, Nawaz M and Ahsan $\mathrm{H}$ : Role of Bcl-2 family proteins and caspases in the regulation of apoptosis. Mol Cell Biochem 351: 41-58, 2011

37. Sha SH, Chen FQ and Schacht J: Activation of cell death pathways in the inner ear of the aging CBA/J mouse. Hear Res 254: 92-99, 2009

38. Hunter AM, LaCasse EC and Korneluk RG: The inhibitors of apoptosis (IAPs) as cancer targets. Apoptosis 12: 1543-1568, 2007. 